\title{
An In Vitro Comparison of Antimicrobial Effects of Curcumin-Based Photodynamic Therapy and Chlorhexidine, on Aggregatibacter actinomycetemcomitans
}

\author{
Shamsoulmolouk Najafi ${ }^{1,2}$, Mina Khayamzadeh' ${ }^{1}$, Mojgan Paknejad ${ }^{3}$, Golfam Poursepanj ${ }^{4}$, \\ Mohammad Javad Kharazi Fard ${ }^{4}$, Abbas Bahador ${ }^{5^{*}}$ \\ ${ }^{1}$ Department of Oral Medicine, International Campus, School of Dentistry, Tehran University of Medical Sciences, \\ Tehran, Iran \\ ${ }^{2}$ Department of Dental Research Center and Oral Medicine, School of Dentistry, Tehran University of Medical \\ Sciences, Tehran, Iran \\ ${ }^{3}$ Department of Periodontics, School of Dentistry, Tehran University of Medical Sciences, Tehran, Iran. \\ ${ }^{4}$ International Campus, School of Dentistry, Tehran University of Medical Sciences, Tehran, Iran \\ ${ }^{5}$ Department of Microbiology, School of Medicine, Tehran University of Medical Sciences, Tehran, Iran
}

\section{*Correspondence to Abbas Bahador, PhD; Department of Microbiology, School of \\ Medicine, Tehran University of Medical Sciences, Tehran, Iran. Tel: +982164053210; Fax: +982188955810 Email:abahador@sina.tums.ac.ir}

Published online 7 January 2016

\begin{abstract}
Introduction: Considering the importance of prevention in periodontal diseases and the important role of Aggregatibacter actinomycetemcomitans in induction and progression of these diseases, the aim of the present in vitro study was to compare the antimicrobial effects of chlorhexidine digluconate (CHX), curcumin and light-emitting diode (LED) laser, on this bacterium.

Methods: Antimicrobial activity of curcumin (5 mg/ml), CHX (2\%), LED (120 J/cm²) and LED + curcumin $\left(120 \mathrm{~J} / \mathrm{cm}^{2}+2.5 \mathrm{mg} / \mathrm{ml}\right)$ against $A$. actinomycetemcomitans were tested in vitro, using micro-broth dilution test. One-way analysis of variance (ANOVA) and Tukey's HSD tests served for statistical analysis.

Results: Regarding the minimum inhibitory concentration (MIC), $\mathrm{CHX}$ had a significantly lower MIC than curcumin $(P<0.05)$. Sorted out by bacterial growth from lowest to highest, were CHX, LED + curcumin, curcumin, and LED groups. All the differences were found to be statistically significant $(P<0.05)$ except for the LED group.

Conclusion: We conclude that curcumin is an effective substance in preventing the growth of $A$. actinomycetemcomitans, whose impact is reinforced when used simultaneously with photodynamic therapy (PDT).

Keywords: Aggregatibacter actinomycetemcomitans; Chlorhexidine; Curcumin; Photodynamic therapy.
\end{abstract}

\section{Introduction}

Periodontal diseases are referred to as inflammatory processes in periodontal tissues in response to the aggregation of bacteria on the teeth. The aggregation rarely leads to evident infection, but the inflammatory response caused in the gingival tissue is responsible for the gradual loss of dental collagen's attachment to the alveolar bone. Left overlooked, this phenomenon might end in increased dental mobility and eventually edentulism. ${ }^{1}$ Production of destructive metabolites by gram negative and positive bacteria of the microbial plaque in the oral cavity causes gingivitis, which can play a key role in the progression of inflammation to periodontal diseases. ${ }^{2}$

Aggregatibacter actinomycetemcomitans is an immobile microaerophilic, facultative anaerobic, gram negative coccoid rod, ${ }^{3}$ strongly associated with pathogenesis of periodontal diseases, particularly aggressive generalized periodontitis. ${ }^{4}$ Some of this bacterium's membranous proteins play an important role in attachment to and penetration in tissue cells. ${ }^{5}$ Other virulence factors secreted by this bacterium include an agent that inhibits the reproduction of fibroblasts and a leukotoxin that kills the leukocytes. ${ }^{6}$

Despite the many complications caused by periodontitis, no treatment protocol is known to be able to fully control this disease. Currently, a combination of mechanical treatments and systemic antibiotics are used as treatment ${ }^{7}$; however, periodontal pathogens are not completely removed by the mechanical removal of the biofilm; and there are problems associated with the use of antibiotics,

Please cite this article as follows: Najafi S, Khayamzadeh M, Paknejad M, Poursepanj G, Kharazi Fard MJ, Bahador A. . An in vitro comparison of antimicrobial effects of curcumin-based photodynamic therapy and chlorhexidine, on Aggregatibacter actinomycetemcomitans. J Lasers MedSci.2016;7(1):21-25.doi:10.15171/jlms.2016.05. 
including weak compliance of patients, ${ }^{8}$ difficulty maintaining therapeutic antimicrobial concentrations in the periodontal plaque, ${ }^{9}$ and eventual complications related to antibiotics such as allergic reactions, gastrointestinal problems and bacterial resistance. ${ }^{10}$ Therefore, researchers are trying to find alternative methods for treatment of periodontal diseases.

Photodynamic therapy (PDT) has been used mostly in the treatment of tumoral lesions, and is now considered as one of the alternative methods. PDT involves three main components: visible light, a photosensitizer (PS) and oxygen molecules. ${ }^{11}$ In this method the PS, which is a photoactivatable substance selective for the prokaryotic target cells, binds to the cells and is activated by light at a specific wavelength. Free radicals are formed, such as oxygen species that have a toxic effect on cells. ${ }^{11-14}$ PDT is a repeatable procedure which costs less than other methods, has a broad spectrum microbicidal effects, is safe for the host tissue, and can reach inaccessible sites. ${ }^{15}$

Another alternative method to treat periodontal diseases is to find substances with anti-plaque effects that can inhibit bacterial reproduction. Curcumin is one of these potential substances, derived from the rhizome of Curcuma longa, which has been reported to show anti-inflammatory, ${ }^{16}$ anti-oxidant, ${ }^{17}$ anti-microbial ${ }^{18}$ and wound healing ${ }^{19}$ properties. The first three mentioned properties make curcumin a favorable substance for the treatment of periodontal diseases. Other than its direct effects, curcumin has also been evaluated as a photosensitizer for PDT. It has a rather broad absorption peak in the 300 to $500 \mathrm{~nm}$ range and shows no toxic effects in a number of cell cultures and animal studies, which makes it a suitable candidate as a PS. ${ }^{20}$

Considering the importance of prevention in periodontal diseases and the important role of A. actinomycetemcomitans in their induction and progression, the aim of the present in vitro study was to compare the antimicrobial effects of curcumin and light-emitting diode (LED) laser on this bacterium, with chlorhexidine digluconate (CHX), as the gold standard mouthwash.

\section{Methods}

Bacterial Strain and Culture Conditions

Lyophilized A. actinomycetemcomitans (ATCC cultures 33384) were obtained from Rayen Biotechnology Co. Ltd., Tehran, Iran. The bacteria were rehydrated in brain heart infusion (BHI) broth (Merck, Darmstadt, Germany); and incubated in a microaerophilic atmosphere at $37^{\circ} \mathrm{C}$ for 48 hours. For assays requiring cultures on plates, cultures grown in supplemented BHI broth were transferred onto BHI agar (Merck, Darmstadt, Germany) plates. Fresh BHI bacterial culture, in the logarithmic growth phase, was adjusted to a concentration of $10^{6} \mathrm{CFU}$ (colony-forming units)/ml as verified by colony counting.

\section{Materials}

Curcumin (Sigma-Aldrich, South Korea) (5 mg/ml) was dissolved in dimethyl sulfoxide (DMSO; Sigma-Aldrich,
South Korea), just before each treatment. ${ }^{20}$ The final concentration of DMSO in the medium was $0.5 \%$. Solutions with a concentration of $2 \% \mathrm{CHX}$ were prepared by diluting 20\% CHX (Sigma-Aldrich, South Korea) into the appropriate concentration with purified water.

\section{Light Source}

A blue light LED system (DB-685 Penguin, COXO, China) with a real intensity of $400 \mathrm{~mW} / \mathrm{cm}^{2}$ and a wavelength of 420-480 nm was applied for 5 minutes (energy density of $120 \mathrm{~J} / \mathrm{cm}^{2}$ ), in PDT producer.

\section{Minimum Inhibitory Concentration}

A broth microdilution method was used to determine the minimum inhibitory concentration (MIC) of curcumin and CHX against $A$. actinomycetemcomitans, as described previously. ${ }^{17}$ Briefly, testing was done using 96well flat-bottomed microplate (Nunc, Denmark), with an assay volume of $200 \mu \mathrm{l} /$ well. First, Mueller-Hinton broth (MHB) (Merck, Darmstadt, Germany) was added (90 $\mu \mathrm{l})$ to each well. The curcumin $(5 \mathrm{mg} / \mathrm{ml})$ and $\mathrm{CHX} 2 \%$ were added (90 $\mu \mathrm{l} /$ well) to wells and serially diluted two-fold across the plate. The plates were then inoculated with a 20 $\mu \mathrm{l} /$ well of fresh MHB bacterial cultures, with a concentration of $10^{6} \mathrm{CFU} / \mathrm{ml}$ for $A$. actinomycetemcomitans. The final bacterial cell concentration in the wells was $10^{5} \mathrm{CFU} /$ $\mathrm{ml}$. Then the microplates were incubated for 48 hours at $37^{\circ} \mathrm{C}$, under microaerophilic conditions. The MIC was defined as that concentration of the substance that will inhibit the visible growth of microorganism after 48 hours of incubation.

To determine whether LED alone caused any reduction of cell viability, bacterial suspensions were exposed to LED at 1, 3, and $5 \mathrm{~min}$ (energy density of 24, 72 and $120 \mathrm{~J} /$ $\mathrm{cm}^{2}$ ), as described previously. ${ }^{17}$ The lowest of LED exposure time that prevented bacterial growth was considered to be the minimum inhibitory LED irradiation dose. All tests were repeated at least 3 times.

Antimicrobial Effect of Treatments Against A. actinomycetemcomitans

To determine the antimicrobial effect of treatments on A. actinomycetemcomitans, samples were distributed to 5 groups as follow: (1) Groups $\mathrm{C}^{+} \mathrm{L}^{+}$(treated with MIC of curcumin and LED), (2) Group $\mathrm{C}^{+} \mathrm{L}^{-}$(treated only with MIC of curcumin), (3) Group $\mathrm{CH}^{+} \mathrm{L}^{-}$(treated only with MIC of $\mathrm{CHX}$ ), (4) Group $\mathrm{C}^{-} \mathrm{CH}^{-} \mathrm{L}^{+}$(treated only with LED), and (5) Group $\mathrm{C}^{-} \mathrm{CH}^{-} \mathrm{L}^{-}$(negative control; no exposure to either curcumin, CHX or LED light). The C, $\mathrm{CH}$ and L indicate "curcumin," "CHX" and "light," respectively. Tests were performed using previously described procedure. ${ }^{17}$ For each group the experiment was repeated 12 times.

\section{Statistical analysis}

SPSS version 20 software served for statistical analysis. One-way analysis of variance (ANOVA) was used to analyze the differences between means, and the post hoc test 
of Tukey's HSD served to evaluate the significance of all paired groups comparisons, with a $P$ value of less than 0.05 considered as statistically significant.

\section{Results}

Based on the results obtained for the MIC of the experimental substances, CHX had the lowest MIC, which was $0.12 \%$. The MIC measured for curcumin was $2.5 \mu \mathrm{g} / \mathrm{ml}$. Our data revealed no effect of LED intensity up to $120 \mathrm{~J} /$ $\mathrm{cm}^{2}$ on the survival of $A$. actinomycetemcomitans.

No growth was observed in our negative control groups. Table 1 shows the logarithm of the CFU/ml of bacteria in all 12 experiments of each group. Descriptive statistics of these results are shown in the Table 2.

As it can be seen in Table 2, the mean of the 12 experiments is the lowest in CHX group with 0.93. After that in the second place with the highest potency and least calculated mean is the LED + curcumin group, with 5.56. Groups curcumin, LED and the control group follow the first two mentioned groups with 6.89, 8.1 and 8.5, respectively. The differences between these figures were evaluated by one-way ANOVA and the post hoc test of Tukey's HSD, the results of which are presented in Table 3.

According to these results, CHX had the greatest potency with significant differences compared to other groups. Simultaneous use of LED with curcumin was found to have the highest potency after CHX, which was also statistically different from the other groups. Following them were curcumin, LED and the control group. The analysis showed that the differences between the curcumin group and the LED group were significant, but the LED group was insignificantly different from the control group with a $P$ value of 0.095 .

Table 1. Logarithm of the CFU/ml of the Bacteria for All the 12 Experiments of Each Study Group

\begin{tabular}{llllll}
\hline No. & Control & Curcumin & Curcumin+LED & LED & CHX \\
\hline 1 & 8.90 & 6.56 & 5.39 & 8.26 & 0.84 \\
2 & 8.78 & 7.19 & 6.48 & 8.16 & 1.14 \\
3 & 8.12 & 6.93 & 4.55 & 7.78 & 1.04 \\
4 & 8.19 & 7.28 & 6.12 & 8.01 & 1.36 \\
5 & 8.16 & 6.33 & 5.46 & 7.68 & 1.14 \\
6 & 8.93 & 6.83 & 5.09 & 8.31 & 1.04 \\
7 & 8.87 & 7.65 & 5.82 & 8.42 & 1 \\
8 & 8.02 & 6.45 & 5.61 & 7.71 & 1.04 \\
9 & 8.92 & 6.61 & 6.26 & 7.94 & 0.69 \\
10 & 8.65 & 6.72 & 5.62 & 8.37 & 0.90 \\
11 & 8.23 & 7.62 & 5.01 & 8.11 & 0.50 \\
12 & 8.98 & 6.56 & 5.33 & 8.52 & 1.04 \\
Mean & 8.56 & 6.89 & 5.56 & 8.10 & 0.93 \\
\hline
\end{tabular}

\section{Discussion}

Many studies have been conducted aiming to find alternative treatments to mechanical removal and antibiotic administration for periodontal diseases. Among all the bacteria involved in the etiology of these oral health problems, A. actinomycetemcomitans has an important role in aggressive generalized periodontitis, and therefore has been paid much attention by many researchers. In this survey we aimed to evaluate the effects of curcumin and LED on this specific bacterium by comparing them to $\mathrm{CHX}$, currently used worldwide. The overall results yielded from our survey were promising; but as could be expected, the MIC results showed greater preventive potency of CHX compared to curcumin on bacterial growth. The antimicrobial effects of curcumin and LED laser have been evaluated on different strains of bacteria. In 2013, Paschoal et $\mathrm{al}^{21}$ evaluated this preventive method on Streptococcus mutans in a planktonic culture. They found that simultaneous use of blue light LED and curcumin had better antibacterial effect on $S$. mutans. Their results were congruent with ours. We worked on A. actinomycetemcomitans since it seems to be a more important bacterium considering its complications. We also aimed to compare the efficacy of this method with the use of each component alone, and we found that simultaneous application of curcumin and LED laser inhibited the growth of $A$. actinomycetemcomitans more efficiently than using curcumin or LED laser individually. Another survey by Mattiello et $\mathrm{al}^{22}$ assessed the effectiveness of $0.01 \%$ toluidine blue accompanied by diode laser of Aluminum Gallium Indium Phosphorus (AlGaInP), on A. actinomycetemcomitans. They also found a positive effect of PDT on this bacterium, which was compatible with our results. They evaluated a synthetic substance whose application might cause various complications. Thus, we chose curcumin, a natural derivative of medicinal plants, with fewer complications expected.

Abdul Azeez et $\mathrm{al}^{23}$ also mentioned in 2014, the synergistic effect of visible blue light emitted from a light-cure laser and 0.2\% CHX mouthwash against A. actinomycetemcomitans. Since oral complications are observed in chronic use of $\mathrm{CHX}$, it is more favorable to find natural substances with fewer side-effects. Thus, we aimed to evaluate the effects of curcumin. In the same year Moslemi et $\mathrm{al}^{24}$ conducted a study on the antimicrobial effects of Radachlorin with PDT on A. actinomycetemcomitans. They also reported the synergistic effects, due to a simultaneous use of these methods. Moreover, they found that Radachlorin had a greater inhibitory effect on the growth of these bacteria, compared to toluidine blue. They also

Table 2. Descriptive Statistics of the Mean of the Logarithm of Bacteria's CFU/ml in the Study Groups

\begin{tabular}{lcccccc}
\hline Study group & N & Mean & SE & SD & Range & 95\% CI for Mean \\
\hline Curcumin & 12 & 6.8942 & 0.12880 & 0.44619 & $6.33-7.65$ & $6.6107-7.1777$ \\
LED+curcumin & 12 & 5.5617 & 0.15952 & 0.55259 & $4.55-6.48$ & $7.2106-5.9128$ \\
LED & 12 & 8.1058 & 0.08208 & 0.28433 & $7.68-8.52$ & $0.00-1.36$ \\
CHX & 12 & 0.9358 & 0.09760 & 0.33811 & $0.7210-1.1507$ \\
Control & 12 & 8.5625 & 0.11017 & 0.38163 & $8.02-8.98$ & $8.3200-8.8050$ \\
\hline
\end{tabular}


Table 3. The Results of Tukey's HSD Test

\begin{tabular}{lccccc}
\hline \multirow{2}{*}{ Group } & N & \multicolumn{4}{c}{ Subset for Alpha = 0.05 } \\
\cline { 3 - 6 } & 12 & 0.9358 & $\mathbf{2}$ & $\mathbf{3}$ & $\mathbf{4}$ \\
\hline $\mathrm{Ch}$ & 12 & & 5.5617 & & \\
$\mathrm{Cu} / \mathrm{LED}$ & 12 & & & 6.8942 & \\
$\mathrm{Cu}$ & 12 & & & & 8.1058 \\
$\mathrm{LED}$ & 12 & & & & 8.5625 \\
Control & & 1.000 & 1.000 & 1.000 & .095 \\
$P$ value & & & & & \\
\hline
\end{tabular}

focused on synthetic materials, whose effects in vivo should be carefully evaluated for eventual complications or side-effects.

The antibacterial effect of curcumin and LED laser on other bacteria including S. mutans and Lactobacillus acidophilus was assessed in a study conducted by Araújo et al in $2014 .{ }^{25}$ They also reported the same results indicative of a better effect in a simultaneous use of these methods. As mentioned before, the more aggressive complications of $A$. actinomycetemcomitans make this bacteria a more important target in research, which was focused on in our survey.

Other than the in vitro experiments mentioned, researchers have also assessed these methods in vivo. Waghmare et $\mathrm{al}^{26}$ evaluated the effects of CHX and curcumin mouthwashes in prevention of plaque formation and inflammation in 2011. Their results suggested these mouthwashes as effective complementary measures alongside mechanical removal for periodontal diseases, showing a comparable effect between curcumin and $\mathrm{CHX}$. Behal et $\mathrm{al}^{27}$ assessed the effect of a $2 \%$ gel form of curcumin on periodontal problems and they found a significant decrease in plaque formation and inflammation, and proposed this method as a complementary treatment for scaling and root planning.

In a study conducted by Suhag et $\mathrm{al}^{28}$ in 2007 , the effect of curcumin as a subgingival irrigator was evaluated, which showed promising results compared to $\mathrm{CHX}$ and normal saline. ${ }^{28}$ A significant decrease was observed in bleeding on probing and gingival redness in the patients treated with curcumin irrigator in the first days of treatment, while the differences with chlorhexidine and normal saline were not significant in the later recall visit. These three studies failed to compare the effects of curcumin and $\mathrm{CHX}$ with simultaneous application of these materials with LED laser. Also in vivo experiments such as these cannot evaluate the effects of a substance on a specific bacterium.

Our results pointed out the effectiveness of curcumin on preventing the growth of A. actinomycetemcomitans, which was reinforced when used simultaneously with PDT.

In this survey, we only evaluated the effects of mentioned methods on one specific strain of bacteria, which can be referred to as one of the limitations in our study. Also we did not assess the effects of various concentrations of substances or doses of radiation or even radiations with different wavelengths and durations. The most important part of the researches aiming to find substitute treatments should be conducted on in vivo environments, so that the complications are discovered as well as the positive effects. So putting it all together, further investigations are required to cover all the limitations of our survey.

\section{Conclusion}

It can be concluded that curcumin is an effective substance in preventing the growth of $A$. actinomycetemcomitans, whose impact is reinforced when used simultaneously in PDT procedure.

\section{Conflict of Interest}

The author has no conflict of interest to declare.

\section{References}

1. Socransky S, Haffajee A, Smith C, Dibart S. Relation of counts of microbial species to clinical status at the sampled site. J Clin Periodontol. 1991;18(10):766-775.

2. Madianos P, Bobetsis Y, Kinane D. Generation of inflammatory stimuli: how bacteria set up inflammatory responses in the gingiva. J Clin Periodontol. 2005;32(s6):57-71.

3. Schacher B, Baron F, Rossberg M, Wohlfeil M, Arndt R, Eickholz P. Aggregatibacter actinomycetemcomitans as indicator for aggressive periodontitis by two analysing strategies. J Clin Periodontol. 2007;34(7):566-573.

4. Rêgo RO, Spolidorio DM, Salvador SL, Cirelli JA. Transmission of Aggregatibacter actinomycetemcomitans between Brazilian women with severe chronic periodontitis and their children. Braz Dent J. 2007;18(3):220-4.

5. Fine DH, Markowitz K, Furgang D, Velliyagounder K. Aggregatibacter actinomycetemcomitans as an early colonizer of oral tissues: epithelium as a reservoir? J Clin Microbiol. 2010;48(12):4464-4473. doi:10.1128/JCM.00964-10.

6. Guthmiller J, Lolly E, Korostoff J. Beyond the specific plaque hypothesis: are highly leukotoxic strains of Actinobacillus actinomycetemcomitans a paradigm for periodontal pathogenesis? Crit Rev Oral Biol Med. 2001;12(2):116124.

7. Deas DE, Mealey BL. Response of chronic and aggressive periodontitis to treatment. Periodontology 2000. 2010;53(1):154-166. doi:10.1111/j.16000757.2009.00334.x.

8. Loesche W, Grossman N, Giordano J. Metronidazole in periodontitis (IV). The effect of patient compliance on treatment parameters. J Clin Periodontol. 1993;20(2):96104.

9. Feres M, Haffajee A, Allard K, Som S, Goodson J, Socransky S. Antibiotic resistance of subgingival species during and after antibiotic therapy. J Clin Periodontol. 2002;29(8):724735.

10. SLOTS J, Ting M. Systemic antibiotics in the treatment of periodontal disease. Periodontology 2000. 2002;28(1):106176.

11. Ribeiro DG, Pavarina AC, Dovigo LN, et al. Photodynamic inactivation of microorganisms present on complete dentures. A clinical investigation. Lasers Med Sci. 2012;27(1):161-168. doi:10.1007/s10103-011-0912-3.

12. Novaes AB Jr, Schwartz-Filho HO, de Oliveira RR, Feres M, Sato S, Figueiredo LC. Antimicrobial photodynamic therapy in the non-surgical treatment of aggressive periodontitis: microbiological profile. Lasers Med Sci. 2012;27(2):389- 
395. doi:10.1007/s10103-011-0901-6.

13. Pinheiro SL, Donegá JM, Seabra LM, et al. Capacity of photodynamic therapy for microbial reduction in periodontal pockets. Lasers Med Sci. 2010;25(1):87-91. doi:10.1007/ s10103-009-0671-6.

14. Calin MA, Parasca S. Light sources for photodynamic inactivation of bacteria. Lasers Med Sci. 2009;24(3):453-460. doi:10.1007/s10103-008-0588-5.

15. Konopka K, Goslinski T. Photodynamic therapy in dentistry. J Dent Res. 2007;86(8):694-707.

16. Satoskar R, Shah S, Shenoy S. Evaluation of anti-inflammatory property of curcumin (diferuloyl methane) in patients with postoperative inflammation. Int J Clin Pharmacol Ther Toxicol. 1986;24(12):651-654.

17. Masuda T, Jitoe A, Isobe J, Nakatani N, Yonemori S. Anti-oxidative and anti-inflammatory curcumin-related phenolics from rhizomes of Curcuma domestica. Phytochemistry. 1993;32(6):1557-1560.

18. Negi P, Jayaprakasha G, Jagan Mohan Rao L, Sakariah K. Antibacterial activity of turmeric oil: a byproduct from curcumin manufacture. J Agric food Chem. 1999;47(10):42974300.

19. Panchatcharam M, Miriyala S, Gayathri VS, Suguna L. Curcumin improves wound healing by modulating collagen and decreasing reactive oxygen species. Mol Cell Biochem. 2006;290(1-2):87-96.

20. Haukvik T, Bruzell E, Kristensen S, Tønnesen H. Photokilling of bacteria by curcumin in selected polyethylene glycol 400 (PEG 400) preparations. Studies on curcumin and curcuminoids, XLI. Pharmazie. 2010;65(8):600-606.

21. Paschoal MA, Tonon CC, Spolidório DM, Bagnato VS, Giusti JS, Santos-Pinto L. Photodynamic potential of curcumin and blue LED against Streptococcus mutans in a planktonic culture. Photodiagnosis Photodyn Ther.
2013;10(3):313-319. doi:10.1016/j.pdpdt.2013.02.002.

22. Mattiello FD, Coelho AA, Martins OP, Mattiello RDL, Ferrão Júnior JP. In vitro effect of photodynamic therapy on Aggregatibacter actinomycetemcomitans and Streptococcus sanguinis. Braz Dental J. 2011;22(5):398-403.

23. Abdul Azeez AR, Mahmood MS, Mahmood W, et al. The effect of chlorhexidine mouth wash and visible blue light on Aggregatibacter actinomycetemcomitans and Porphyromonas gingivalis of patients with chronic periodontitis (an in-vitro study). IOSR Journal of Dental and Medical Sciences. 2014;13(7):1-7.

24. Moslemi N, Soleiman-Zadeh Azar P, Bahador A, et al. Inactivation of Aggregatibacter actinomycetemcomitans by two different modalities of photodynamic therapy using Toluidine blue $\mathrm{O}$ or Radachlorin as photosensitizers: an in vitro study. Lasers Med Sci. 2014;30(1):89-94. doi:10.1007/ s10103-014-1621-5.

25. Araújo N, Fontana C, Bagnato V, Gerbi M. Photodynamic antimicrobial therapy of curcumin in biofilms and carious dentine. Lasers Med Sci. 2014;29(2):629-635. doi:10.1007/ s10103-013-1369-3.

26. Waghmare P, Chaudhari A, Karhadkar V, Jamkhande A. Comparative evaluation of turmeric and chlorhexidinegluconate mouthwash in prevention of plaque formationand gingivitis: a clinical and microbiological study. J Contemp Dent Pract. 2011;12(4):221-224.

27. Behal R, Mali AM, Gilda SS, Paradkar AR. Evaluation of local drug-delivery system containing $2 \%$ whole turmeric gel used as an adjunct to scaling and root planing in chronic periodontitis: A clinical and microbiological study. J Indian Soc Periodontol. 2011;15(1):35-38.

28. Suhag A, Dixit J, Dhan P. Role of curcumin as a subgingival irrigant: a pilot study. Perio. 2007;4(2):115-121. 